Review

\title{
Quantum Dots: An Insight and Perspective of Their Biological Interaction and How This Relates to Their Relevance for Clinical Use
}

\author{
Martin J. D. Clift ${ }^{1 凶}$ and Vicki Stone ${ }^{2}$ \\ 1. Bionanomaterials, Adolphe Merkle Institute, University of Fribourg, Rte de l'Ancienne Papeterie, Marly 1, 1723, Fri- \\ bourg, Switzerland. \\ 2. Nanosafety Research Group, School of Life Sciences, Heriot-Watt University, Edinburgh, EH14 4AS, United Kingdom.
}

$\checkmark$ Corresponding author: Martin J. D. Clift, BioNanomaterials, Adolphe Merkle Institute, University of Fribourg, Rte de l'Ancienne Papeterie, Marly 1, 1723, Fribourg, Switzerland. Tel \#: 00412630095 17, Fax \#: 0041263009624 , e-mail: martin.clift@unifr.ch.

(C) Ivyspring International Publisher. This is an open-access article distributed under the terms of the Creative Commons License (http://creativecommons.org/ licenses/by-nc-nd/3.0/). Reproduction is permitted for personal, noncommercial use, provided that the article is in whole, unmodified, and properly cited.

Received: 2012.05.02; Accepted: 2012.07.19; Published: 2012.07.25

\begin{abstract}
Due to their novel physico-chemical characteristics, semi-conductor nanocrystal quantum dots (QDs) provide an advantageous perspective towards numerous different consumer and medical applications. The most notable potential application of QDs is their use as therapeutic and diagnostic tools in nanomedicine. Despite the many benefits posed by QDs, the proposed, intentional exposure to humans has raised concerns towards their potential impact upon human health. These concerns are predominantly based upon the heterogeneous composition of QDs, which most commonly comprises of a cadmium-based core and zinc sulphide shell. Whilst other nanoparticle (NP) types possess a similar structure to QDs (i.e. core-shell technology (e.g. $\mathrm{Fe}_{2} \mathrm{O}_{3}, \mathrm{Au}$ and superparamagnetic iron oxide NPs)), the importance of the concerns surrounding human exposure to QDs is amplified further since, due to the sophisticated chemical and light-emitting properties of QDs, the use of these NPs within any (nano)medical setting/application could be suggested as realistic, rather than simply an advantageous possibility. It is therefore imperative that a thorough understanding of how QDs interact with various biological systems, predominantly those relative to humans and what the consequences of such interactions are is gained with extreme alacrity. It is the aim of this review to highlight the current knowledge base of QD-biological system interactions, where the knowledge gaps (still) remain and how the understanding of this interaction relates to the most notable of applications for QDs; their clinical relevance.
\end{abstract}

Key words: Quantum dots; Nanoparticles; Nanomedicine; Nanocrystals; NP-cell Interactions; Human Health Effects; Clinical Relevance.

\section{Introduction}

Semi-conductor nanocrystal quantum dots (QDs) are a prime example of a nanoparticle (NP); defined as a nano-object [a material with one, two or three external dimensions in the nanoscale (1-100 $\mathrm{nm})$ ] with all three external dimensions in the nanoscale ${ }^{1}$; that is intended for use in nanomedicine ${ }^{2}$. Whilst a plethora of alternative, hybrid NPs are also available that exhibit similar properties to QDs, such as $\mathrm{Fe}_{2} \mathrm{O}_{3}$ or $\mathrm{Au}$ NPs with an incorporated polymer shell, as well as superparamagnetic iron oxide NPs $(\mathrm{SPIONs})^{3}$, which are easily synthesised with respect to 'core-shell' technology, QDs are unique in their characteristics exhibiting a narrow excitation/ emission wavelength, as well as increased fluores- 
cence and photostability which enables them to be ideal tool for many biomedical applications, such as therapeutics and diagnostics ${ }^{4,5}$.

As with all nano-based materials or NPs, QDs have received a heightened degree of attention in the last two decades ${ }^{6}$ due to their physico-chemical characteristics and as to what their potential adverse effects may be following their, inevitable, exposure to humans ${ }^{7}$. The intended application of QDs suggests that only intentional exposure, via injection, would be a potential risk to humans and their health. Whilst this is undoubtedly correct under the assumption of QDs being applied within a clinical setting, the notion of risk (hazard $x$ exposure) is also perceived through the manufacturing of QDs, and thus the possibility of the inhalation of QDs by humans is a realistic problem that requires immediate attention. It is also important to point out that, considering the major exposure routes of NPs to the human body ${ }^{8}$, QDs may also pose a risk towards both the skin and the gastro-intestinal tract. It is difficult to understand the notion as to why QDs may be of risk to the gastro-intestinal tract, unless (i) they are used as a 'drug-device' via oral administration, which is not the primary intention for the application of QDs within nanomedicine, or (ii) they locate in this region following injection and or inhalation. It is highly unlikely that the latter could occur however, since if the QDs, or NPs in general, are to translocate to an (another) organ following their administration (either via inhalation or injection), they would most likely locate within other organs ${ }^{9}$, such as the liver ${ }^{10}$. Furthermore, following such an 'exhaustive' translocation process it is debatable, despite their suggested heightened stability, as to whether or not the QDs would still be 'complete' QDs, and not simply fragments of the original NP itself; this aspect is beyond the scope of the present review, but is well discussed within the article of Geys et al. ${ }^{11}$ as is becoming an ever increasing research scope within the field of nanotoxicology ${ }^{12}$. This of course highlights an ongoing issue regarding the translocation and subsequent 'secondary toxicity' of NPs in the human body - an area that is receiving increased attention and investigation ${ }^{9}$. In addition to this, there has also been increased research into the effects of QDs on the skin $^{13-15}$. Whilst such research is important to conduct in regards to gaining a thorough risk assessment ${ }^{16}$ of any NP type, the applicability of such a research outlook towards the specific and intended application of QDs does not warrant immediate or imperative investigation compared to how QDs may affect cells relative to either the human lung or bloodstream. It is also prudent to highlight that research into the effects of QDs on blood cells (i.e. red blood cells or platelets) is lacking. This is true for all NPs in regards to their effects on human blood cells. Although much of the research within the field of nanotoxicology and the effects of NPs on human health focusses upon the immune system, little has actually delved into the effects of how human blood cells 'see' NPs. Of the limited research conducted (e.g. $\left.{ }^{17}\right)$, extremely interesting findings have been reported. Such as the notion that red blood cells can internalise NPs of $<100 \mathrm{~nm}$ despite the fact that they express not phagocytic properties ${ }^{18}$. Furthermore, many studies have assessed the haemolytic potential of NPs. The haemolysis assay, although dated, is still considered an adequate and representative model biochemical protocol to determine how xenobiotics may affect red blood cells ${ }^{19}$. This assay therefore, is especially advantageous as a short-term in vitro assay to determine NP toxicology ${ }^{20}$. In the study of $\mathrm{Lu}$ and colleagues, the haemolysis assay was used to assess the effects of a series of metal oxide NPs, including alumina, magnesium, silica, zinc, copper and nickel. Whilst the majority of metal oxides assessed showed no haemolysis, exposure of cerium dioxide and a form of alumina, in addition to nickel resulted in a significantly elevated haemolytic effect upon human red blood cells ${ }^{21}$. Also, a recently study by Zhu et al. ${ }^{22}$, the effects of anionic polyurethane NPs were assessed for their platelet activation and haemolytic capability. In fact, a number of different particle types, such as hydroxyapatite and tricalcium phosphate NPs ${ }^{23}$ and iron oxide $\mathrm{NPs}^{24}$ have been assessed for their interaction with human blood by using the haemolysis assay. In addition to this, recent research using aqueous synthesized QDs with a Cd core has shown that QDs can have a blood circulation time, as determined by serum biochemistry, of between 15-80 days ${ }^{25}$. Su and colleagues ${ }^{25}$ reported that the aqueous QDs also located within the liver of female Balb/c mice after 0.5 to 4 hours, although no significant toxic (or lethal) effects were observed. Despite such findings however, and considering the primary intended application of QDs, an understanding of how QDs may 'interact' with the human body is limited.

\section{Why is there a limited understanding of the biological interaction of QDs?}

Despite the benefits of QDs posing a considerable advantage to the potential clinical advancement in the identification and treatment of diseases (i.e. tumour formation) $)^{2,4,26}$, concerns as to the human exposure of QDs have been raised based upon both (i) the surface properties (associated with the size and shape) of QDs and (ii) the materials used to construct these core-shell NPs. Usually, these highly fluorescent 
NPs range in size (core) from $1 \mathrm{~nm}$ to $25 \mathrm{~nm}$ in diameter and their fluorescent properties are strongly correlated to their size, which, in terms of QDs, also corresponds to their shape (in regards to a change in the diameter of the QD core) $)^{2,26}$. Also, the increased anxiety associated with the potential risk associated with human exposure to QDs is due to the fact that QDs consist of a 'toxic' core material; commonly either cadmium telluride (CdTe) or cadmium selenide (CdSe), , and are covered with a shell coating, such as zinc sulphide $(\mathrm{ZnS})^{4}$. Although it is suggested that such a shell coating is 'biocompatible' 27 , the presence of $\mathrm{Cd}$ as a core material, at the nanoscale, exudes the notion that QDs could be 'lethal', and possibly similar to that observed with $\mathrm{Cd}$ in the bulk form. The complexity of QDs therefore, is a major reason as to why the knowledge base surrounding QDs is currently limited - a similar problem concerning all hybrid $\mathrm{NPs}^{3,4}$. It is important to note however, that although $\mathrm{Cd}$ is used as a prominent core material for QDs, there are many other core materials are available with inorganic cores, such as silicon ${ }^{28}$. Whilst it is perceived that such alternative core materials could negate the adverse biological effects (as discussed within this review $)^{29}$, investigation into how such 'alternative-core' QDs impact upon biological systems is in its infancy, and much of what is known regarding the biological interaction of QDs is based on Cd-core QD $\mathrm{NPs}^{30}$, as thus forms a basis within the present review.

Due to the limited understanding within the field therefore, the present review aims to discuss and summarise how the specific physical characteristics of QDs contributes to their biological impact and how these factors relate to their clinical relevance.

\section{Surface Properties of QDs}

Understanding the absolute physico-chemical characteristics of QDs when studying their biological interaction is imperative. From a historical perspective, the surface of NPs has formed the basis for increased research, and is considered to be one of the driving characteristics of why an increased adverse biological effect was first observed following NP exposure ${ }^{12,31,32}$. Whilst the surface area and reactivity ${ }^{33}$ are still considered extremely important characteristics in relation to the biological interaction for any NP type $^{34}$, understanding the key surface characteristics of core-shell NPs, such as QDs is essential to deciphering how they may interact with different biological systems, since changes to the surface properties are suggested to significantly alter the manner in which biological systems may 'observe' NPs ${ }^{35}$, such as the adsorption of proteins ${ }^{36}$ and the kinetic/environmental changes that happen to this ad- sorbed protein corona ${ }^{37}$

Due to these issues, it is imperative that a thorough understanding of the specific physical (surface) characteristics of the NP being investigated are obtained in situ (e.g. in the same buffer and environment (i.e. $\mathrm{pH}$ ) as the biochemical/biological tests are performed $)^{34}$. It is also essential that information pertaining to the NP prior it to being placed in a biological environment. This is particularly relevant to QDs, as well as the many other core-shell NPs, as it is possible to attach a wide variety of biologically-based molecules to their surface, such as proteins, peptides and lipids for cellular targeting2,4,26 (Fig. 1). The use of any of these surface attachments can also have a significant effect on the size of QDs, with the final diameter suggested to be able to range from $1 \mathrm{~nm}$ to $100 \mathrm{~nm}^{4}$. This potential change in diameter of the QDs further emphasises the necessity to understand the basic characteristics of the NP prior to any biological testing, since, depending upon the specific NP type, biological media can promote the agglomeration/aggregation of NPs and thus change the surface properties of any NP38.

Another surface characteristic that is paramount in the synthesis and proposed application is the addition of a polymer coating layer (Fig. 1). Polymer coatings can also be attached to the surface of QDs, and have an additional effect upon their size (up to $>40 \mathrm{~nm}$ in diameter $)^{4,26}$. It is intended that the use of polymer coatings on NP surfaces will form 'stealth' NPs, which will be able to be targeted to specific organs within the body in order to diagnose, treat or prevent disease ${ }^{39,40}$. Many different forms of polymer coatings are possible for use on the surface of QDs, such as poly-vinyl alcohol (PVA), poly-meythl methacrylate (PMMA) and poly-lactide-co-glycolides (PLGA). The most commonly and popular polymer shell used, especially with QDs due to their intended application within nanomedicine, is poly-ethylene glycol (PEG).

The potential benefits of using PEG as a coating on the surface of NPs can be related to previous findings in the drug delivery and drug design literature ${ }^{39,41}$. It has been shown that the addition of a polymer PEG coating on particle-drug complexes is highly advantageous as it promotes a 'steric repulsive barrier' resulting in a lack of recognition by phagocytic cells, responsible for cleaning foreign particles from the blood and tissue ${ }^{42-45}$. It was further reported by Porter et al. ${ }^{2}$ that upon contact with the cell or organ that the site-targeted polymer PEG coating is negated by a specific interaction mechanism between the particle-drug complex and the cell or organ. Research into the effectiveness of PEG as a surface coat- 
ing for nanomedicine has shown it to be advantageous in the targeting ability of cationic lipid-nucleic acid $\mathrm{NPs}^{46}$, as well as demonstrating that this polymeric material is of low toxicity ${ }^{47}$, thus ideal for use on the surface of QDs.

The effects of applying a PEG coating to the surface of QDs have shown similar effects. In a study by Zhang et al. ${ }^{48}$ the toxicity of PEG coating was investigated. It was reported that following the treatment of both lung (IMF-90) and skin (HSF-42) fibroblasts with PEG-silica coated CdSe/ZnS QD with doses of 8 and $80 \mathrm{nM}$, that no significant effects on the cells' ability to undergo mitosis was observed, however a small increase in apoptopic/necrotic cell death was found at both the low and high concentrations respectively. Zhang et al. ${ }^{48}$ also stated that the PEG-silica coated CdSe/ZnS QD showed no inflammatory response or heavy-metal-related toxicity even at $80 \mathrm{nM}$. In addition, Duan and $\mathrm{Nie}^{49}$ also investigated the effects of a PEG surface coating on QDs. It was initially reported that amine surface coated QDs were highly cytotoxic, observed via the MTT assay, after exposure for two hours at $1 \mathrm{nM}$ in Hela cells. Subsequent addition of a PEG/polyethylenimine (PEI) (PEI-g-PEG) surface coating to the amine coated QDs caused the level of toxicity elicited by the amine QDs to significantly decrease at two hours at a concentration of $1 \mathrm{nM}$. Duan and $\mathrm{Nie}^{49}$ further examined the ability of these PEI-g-PEG coated QDs to be taken-up by the Hela cells over two hours. It was observed that spontaneous uptake and intra-cellular localisation did occur over the two hour period. The results of 49 could be further supported by that of Clift et al.50,51 in which it was reported that $\mathrm{NH}_{2}$ (PEG) QDs (both chemical groups were deduced to be equally active on the NP surface) did enter a macrophage cell-line over a period of two hours exposure at 40nM. It was also found that this concentration resulted in a significant depletion in glutathione levels up to $48 \mathrm{hrs}$, although no cytotoxicity, pro-inflammatory marker stimulation or effects upon cellular calcium signalling in vitro ${ }^{52}$ were noted for this form of surface modified QDs.

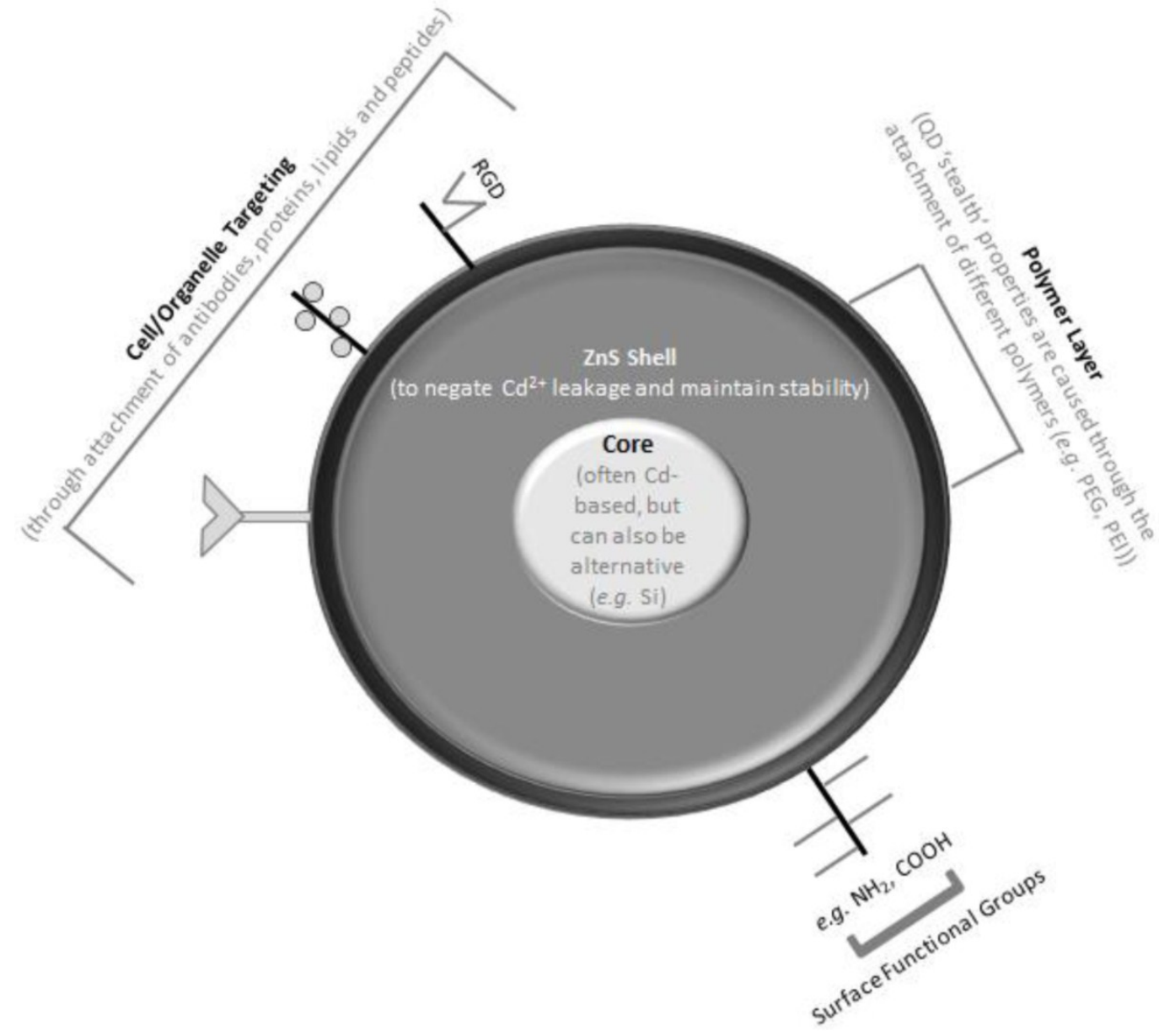

Figure I: Impression of the physical state of a quantum dot (QD) nanoparticle. The figure shows a schematic (not to scale) perspective of the core-shell technology highlighting the different core, shell and polymer materials that can be used to synthesize QDs, as well as the plethora of different modifications that can be adapted to the surface of QDs. 
In summary therefore, whilst a variety of different surface markers/modifications can be easily attached to the surface of QDs (Fig. 1), it is essential that a complete understanding of precisely what is attached to the surface, and how, is gained prior to any biological/biochemical testing strategy is initiated, since it has been proven (as discussed above) that the minimal difference in the (surface) characteristics of QDs can elude to a significantly different biological interaction ${ }^{6}$.

\section{Biological Interactions of QDs}

Since manufactured NPs are predominantly produced from a 'bulk' material via 'top-down' processes, there is constant debate as to whether or not NPs elicit any adverse effects as a result of their material type, or due to their nanoscale properties. This is particularly prominent within the core-shell NP technologies, such as QDs, due to their heterogeneous composition.

\section{Is it only a material effect?}

$\mathrm{Cd}$ is a highly cytotoxic heavy metal and is used within a wide array of applications such as a colour pigment in paints, as well a major ingredient within power batteries ${ }^{53,54}$. This non-essential element can be used in many different forms, such as CdTe and CdSe, as within the core of QDs. Research into the toxicity of bulk $\mathrm{Cd}$ has been based mainly on the effects of $\mathrm{Cd}$ ions $\left(\mathrm{Cd}^{2+}\right) . \mathrm{Cd}^{2+}$ can severely disrupt the function of the mitochondria of cells, particularly in the liver, through inhibiting the oxidative phosphorylation properties of these organelles subsequently causing cancer ${ }^{55}$. In addition to bulk $\mathrm{Cd}$ being a known carcinogen, $\mathrm{Cd}^{2+}$ have also been found to cause a depletion of glutathione, reactive oxygen species (ROS) production and lipid peroxidation in cells ${ }^{56}$. Furthermore, $\mathrm{Cd}^{2+}$ has been found to cause both carcinogenic tumours in vitro and in vivo following exposure, as well as causing cell death via apoptosis $^{54-55,57}$.

It is clear therefore, that the core material of QDs, or the most commonly used core material at least, is adverse towards normal cell homeostasis, and in some instances potential carcinogenic towards humans. Why therefore, can $\mathrm{Cd}$, or $\mathrm{Cd}$ based derivatives, despite being an optimal material (or element) as an image contrast agent (i.e. for diagnostics), be used as the main (core) constituent of QDs?

\section{Can QDs elicit a NP-associated effect?}

In order to gain an insight into this issue, Derfus et al. ${ }^{27}$ originally investigated the possible toxicity of QDs. In this study, Derfus et al. ${ }^{27}$ studied the toxic effects of 'naked' QDs (QDs with no shell material) with a CdSe core and tricytll phosphine oxide (TOPO) surface coating (TOPO capped) on primary rat hepatocyte cells, via the MTT assay. Initially, it was found that these TOPO capped QDs caused no deleterious effects on hepatocyte metabolic competence after exposure for eight hours. Subsequent analysis however, following exposure to ambient air for 30 minutes, as well as UV light for eight hours demonstrated these QDs to significantly decrease hepatocyte cell metabolic activity. A concentration-dependent toxicity was found to occur, which was related to a release of $\mathrm{Cd}^{2+}$ from the QD core. It was further concluded that the release of $\mathrm{Cd}^{2+}$ was due to the oxidation of these NPs within the specific experimental/environmental conditions. Derfus et al. ${ }^{27}$ concluded therefore, that QD toxicity was related to the release of $\mathrm{Cd}^{2+}$ form the core, and that QD toxicity is relative to the specific environmental and experimental conditions in which they are exposed to cells.

It was subsequently hypothesised by Derfus et al. ${ }^{27}$ that the addition of a shell to the QD core, such as zinc sulphide (ZnS) or dihydrolipoic acid, or even a polymer based material, such as polyacrylate, or PEG would reduce the level of oxidation, inhibiting QD toxicity. Although 27 did show that the use of a $\mathrm{ZnS}$ shell negated the decreased level of cell metabolic activity following treatment of hepatocyte cells with TOPO capped QDs these findings did not provide holistic evidence that ZnS could inhibit any QD-associated toxicity. Thus, there is still much debate as to whether or not the $\mathrm{Cd}$ core is responsible for QD toxicity.

Shiohara et al. 58 investigated the cytotoxicity of mercaptoundecanoic acid (MUA) CdSe QDs, which were synthesised in TOPO with sheep serum albumin (SSA), on Vero cells (African green monkey's kidney cells), primary human hepatocytes and Hela cells. It was observed that the Vero cells induced a significant depletion in cell metabolic competence after 24 hours exposure with a series of concentrations up to $10 \mathrm{mg} / \mathrm{ml}$. No effects were shown with either the hepatocyte or Hela cells after this time. Subsequent analysis via flow cytometry, found a dose response to occur in Vero cells, following treatment with these TOPO MUA-SSA capped QDs after 4-6 hours. It was further observed that this dose response induced an increased staining of cells with the fluorescent probe propidium iodide (PI). PI is a marker of cell death and can only enter cells if the membrane is disrupted. Thus it was suggested by Shiohara et al..$^{58}$ that the Vero cells were undergoing necrotic cell death. It was concluded in contrast to Derfus et al. ${ }^{27}$ that this observed cytotoxicity and cell death was due to the 
TOPO MUA-SSA cap on the QDs and not the QD core.

Hoshino et al. ${ }^{59}$ further supported the conclusions of 58 following investigation of the physiochemical properties of a series of five surface modified CdSe core/ZnS shell QDs, including negatively and positively charged QDs. It was observed that negatively charged, $\mathrm{COOH}$ chemical surface coated QDs were less toxic compared to positively charged, amine coated QDs to mouse lymphoma EL-4 cells over 12 hours. It was subsequently concluded by Hoshino et al.60 that the type of QD surface chemistry (coating/charge) is an indicator of their toxicity and not the $\mathrm{Cd}$ core material.

Studies by both Chan et al.61 and Guo et al.62 also refute the conclusions of Derfus et al. ${ }^{27}$. Investigation by Chan et al. ${ }^{61}$ reported TOPO capped CdSe QDs to cause a significant reduction in human neuroblastoma IMR-32 cell metabolic activity (MTT assay) at concentrations of $150 \mathrm{nM}$ and $300 \mathrm{nM}$ after 24 hours. Chan et al.61 subsequently concluded that the observed toxicity caused by the TOPO capped QDs, was due to their organic coating and not the QD core. Additionally, Guo et al. ${ }^{62}$ reported CdSe QDs coated with F-68 CTAB and Sodium Dodecyl Sulfate (SDS) to cause significant cytotoxicity (as assessed via the MTT assay) to HepG2 cells over a 72 hour period. The cytotoxicity observed by Guo et al. ${ }^{62}$ was attributed to the increased toxicity observed with both the F-68 CTAB and SDS surface modifications and not the CdSe core material. Subsequent analysis by Guo et al. ${ }^{62}$, in contrast to the findings of 58 , reported that the F68 CTAB SDS coated CdSe QDs caused heightened toxicity eliciting cell death, comparable to $\mathrm{Cd}$ toxicity, via apoptosis and not necrosis.

In order to determine if the surface charge was responsible for QD toxicity, Lovric et al.63 examined the effects of both green (emission $\lambda-530 \mathrm{~nm}$ ) and red (emission $\lambda-692 \mathrm{~nm}$ ) positively and negatively surface charged 'naked' CdTe QDs in rat pheochromocytoma (PC12) neural and N9 murine microglial cells over a 24 hour period. Initial assessment of their toxicity over 60 minutes observed the green negatively charged QDs to cause no significant effect on cell metabolic activity (using a version of the MTT assay) following treatment for 30 minutes and 60 minutes at concentrations of $1 \mu \mathrm{g} \cdot \mathrm{ml}^{-1}$ to $10 \mu \mathrm{g} \cdot \mathrm{ml}^{-1}$ in rat PC12 cells. Subsequent analysis over 24 hours showed, in comparison to ${ }^{59}$, that the positively charged QDs were greater at reducing the cell metabolic activity (MTT assay) of PC12 and N9 cells than the negatively charged QDs at concentrations of $10 \mu \mathrm{g} \cdot \mathrm{ml}^{-1}$ to $100 \mu \mathrm{g} . \mathrm{ml}^{-1}$. Geys et al. ${ }^{64}$ also assessed the contribution of surface charge towards the biological impact of
QDs. It was observed that carboxylated QDs elicited a heightened negative effect upon male Balb/c mice after $1 \mathrm{hr}$ exposure to $144-3600 \mathrm{p} / \mathrm{mol}$ per mouse via intravenous injection in the tail vein. It was reported that the negatively charged QDs caused an increased thrombosis within the vascular circulation compared to positively charge QDs, although the latter also did show signs of thrombotic injury in vivo. Subsequent analysis, with lower concentrations of QDs (1.44 to $144 \mathrm{p} / \mathrm{mol}$ per mouse) also showed that surface charge played a pivotal role in the biological impact of QDs. Investigation of the dry and wet weight of the animals, as well as the platelet aggregation state and inflammatory state of the animals' lungs (bronchiolar lavage fluid analysis) all showed that, in contrast to Lovric et al.63, although positively charged QDs elicited an altered biological response, the negatively charged QDs caused a significant reduction in the homeostasis of the animals. It was therefore concluded by Geys et al. ${ }^{64}$ that surface charge is a major driving factor regarding QD toxicity. This issue is further described and discussed in detail within the recent review by Hoshino and colleagues ${ }^{65}$.

Whilst Geys et al. ${ }^{64}$ also assessed the Cd content of the lung, liver and blood of the animals (to determine QD concentration), the findings of these studies were not able to determine whether or not the $\mathrm{Cd}$ core or the surface charge was responsible for these toxic effects. In a subsequent study by Clift et al. ${ }^{51}$, the effects of surface charge and each material aspect of QDs (i.e. core only vs. shell only, etc.) were studied. It was observed that organic surface coated QDs (with a TOPO/TOP/Decane organic surfactant layer on the surface) were highly cytotoxtoxic (as assessed via the MTT assay and lactate dehydrogenase release) compared to both negatively $(\mathrm{COOH})$ and positively $\left(\mathrm{NH}_{2}\right.$ (PEG)) QDs at concentrations of $20 \mathrm{nM}$ to $80 \mathrm{nM}$. It was further observed, that the $\mathrm{Cd}$ core material could, if released from the core of the QD, elicit the strongest cytotoxic response compared to the complete QDs and $\mathrm{ZnS}$ shell only. Investigation using inductively coupled-atomic elemental spectroscopy (ICP-AES), found that the amount of $\mathrm{Cd}$ released from organic QDs was insignificant when internalised within cells, however in the absence of cells, or in an environment of $\mathrm{pH} 4.0$ (indicative of a lysosome), a significant $\mathrm{Cd}$ release was observed ${ }^{51}$. The ability for the $\mathrm{Cd}$ core to be released from either the $\mathrm{COOH}$ or $\mathrm{NH}_{2}$ (PEG) QDs was not studied however, since these were not found to be highly cytotoxic. Despite this, since both the $\mathrm{COOH}$ and $\mathrm{NH}_{2}$ (PEG) QDs were found to be located inside lysosomes/endosomes ${ }^{66}$ up to 48 hours after exposure at $40 \mathrm{nM}$ particle concentration, for which this highly acidic environment was observed to con- 
tribute to the decrease in fluorescent stability of these QDs (suggestive of a fragmenting of the NP) ${ }^{50}$, it could be suggested that the Cd core could also be released from these surface functionalised QDs. Thus, to further understand precisely which characteristic of QDs drives their associated adverse effects requires further deduction.

Irrespective of the driving characteristics of QD toxicity, it was further demonstrated by Lovric et al. ${ }^{63}$ however, that QD toxicity increased concomitantly with a decrease in size, with the green QDs found to cause a significantly greater decrease in PC12 cell metabolic competence, suggestive of cell death, compared to the larger red, positively charged QDs after 24 hours exposure. The size of QDs was further found to be specific to the localisation of the NPs inside the cell, with the smaller, green QDs observed to penetrate into the nucleus, possibly causing damage to the cells deoxyribose nucleic acid (DNA) content, and thus attributed as the potential process responsible for the increased cytotoxicity observed. The finding that QD size could be relevant to their toxicity supports previous literature that examined the toxic effects of environmental air pollution particles, suggesting particle size to be a component responsible for NP toxicity12,32, as well as highlighting another characteristic responsible for QD toxicity (in addition to the core and specific surface coating/charge).

Additional investigation by Lovric et al. ${ }^{63}$ examined the effects of pre-treating PC12 cells with antioxidants, specifically $\mathrm{N}$-acetyleysteine, and trolox to determine if the effects observed were mediated via ROS and oxidative stress. It was demonstrated, that pre-treatment of PC12 cells with $\mathrm{N}$-acetyleysteine, at a final concentration of $2 \mathrm{mM}$, and not Trolox or bovine serum albumin (BSA) (both $1 \mathrm{mM}-2.5 \mathrm{mg}^{-\mathrm{ml}^{-1}}$ ) was found to negate any form of cell death to occur, suggesting that this effect may be mediated via ROS production. Subsequent research by Lovric et al.67 showed that 'naked' mercaptopropianic acid (MPA) coated CdTe QDs inhibit human breast cancer (MCF-7) cell function. In addition to the observed reduction in cell metabolic competence (MTT assay), the MPA coated QDs, at concentrations of $5 \mu \mathrm{g} / \mathrm{ml}$ and $10 \mu \mathrm{g} / \mathrm{ml}$, were found to cause increased levels of ROS production in MCF-7 cells following treatment for 30 minutes, as determined by imaging of dihydroethidium (DHE) in MCF-7 cells. Similar to the DCFH-DA reaction in which ROS production is determined by the level of fluorescence, DHE detects the extent of oxidative stress and ROS production in cells, by oxidising to ethidium, which is highly fluorescent. The increased production of ROS was further attributed to the decrease in cell viability, demonstrated via membrane blebbing and decreased cytochrome $\mathrm{c}$ production. Furthermore, the MPA coated QDs were found to be present within the mitochondria of the MCF-7 cells, causing a significant change in morphology, with a reduction in the length observed over the 24 hour period. These findings were attributed therefore, in support of Guo et al.62, to the QDs causing cell death via apoptosis.

Recently, further research into the ability of QDs to produce ROS and cause oxidative stress has been performed by Chan et al.61. As previously mentioned, 63 demonstrated TOPO capped QDs to significantly decrease cell metabolic competence after 24 hours at $150 \mathrm{nM}$ and $300 \mathrm{nM}$ in IMR-32 cells. Chan et al.63 attributed this observed toxicity to an increased production of ROS, assessed via the DCFH method, following treatment for 24 hours at both $150 \mathrm{nM}$ and $300 \mathrm{nM}$ in IMR-32 cells. Using less than $50 \%$ diluted concentrations of those used by 63 , Clift et al52 showed that $\mathrm{COOH}$ and $\mathrm{NH}_{2}$ (PEG) QDs could cause an oxidative stress environment, measured via the decrease in the intracellular thiol glutathione (GSH), in J774.A1 macrophage cells after 48 hours exposure at 20, 40 and 80nM particle concentrations. The organic QDs used in the studies of Clift et al.50,51-52,66 were also assessed for their ability to cause a loss in GSH levels over time. However, due to the nature of their increased cytotoxicity, as previously mentioned, any decrease in GSH content was associated with this characteristic. It was though found that organic QDs could alter the intracellular calcium signalling, similar to that of ultrafine carbon black (ufCB), in J774.A1 cells as measured by the FURA2-AM method. Whilst this observation could also be associated with their cytotoxicity, the notion that QDs could elicit similar effects to ufCB, a surrogate for air pollution particulate, is intriguing and requires in-depth analysis to determine if other similarities do exist.

In summary, the effects of QDs are, in fact, well studied, however only a few clear observations can be thought of as consistent. The most notable of which is the fact that an organic coating can elicit heightened adverse effects irrespective of their other surface characteristics. The understanding as to whether or not it is the core, the material, or the QD itself that drives the adverse effects reported is equivocal, but it seems to be dependent upon the specific QD used and what environment it locates. It is apparent however, that problems lay in determining precisely how apt QDs are for clinical purposes, since many different surface coatings, functionalisations and sizes of QDs have been studied with no clear outline as to what types of surface coatings, functionalisations and sizes are intended for actual use in a clinical setting. 
Despite these differences, it is also prudent to highlight that whilst many of the subsequent biological/biochemical effects have been investigated and published, many suggest that the manner in which the QD 'interacts' with the cell, and where the QDs locate could drive such effects (i.e. ${ }^{63}$ ); a notion that is well accepted already within the field of nanotoxicology ${ }^{68}$. Therefore, what is known about the actual 'interaction' (i.e. the uptake mechanisms and intracellular localisation) of cells and QDs?

\section{How do QDs 'interact' with biological systems?}

As previously mentioned, Lovric et al.63 suggested that the location of QDs could be pertinent to the toxic and adverse cellular responses associated with these highly fluorescent NPs. Despite this, an understanding of the actual cellular process of the uptake and sub-cellular localisation and distribution of QDs is limited. As previously highlighted in the present review, as well as in reviews by ${ }^{6,69-70}$ it was concluded that the surface coating has a significant role in the biological interaction of QDs, and thus in the cellular uptake and intracellular localisation of QDs.

Examination of the uptake processes and sub-cellular localisation of QDs, has found that avidin-conjugated CdSe/ZnS QDs undergo endocytosis into endosomes within in eukaryotic cells following live cell laser scanning confocal microscopy ${ }^{71}$. Also, investigation of CdSe/ZnS QDs within a michelle/polymer coating has found that these NPs are able to penetrate the nucleus, similar to Lovric et al.63, of 'Xenopus' embryos, however cause no toxicity when present within these frog embryos for up to two hours ${ }^{72}$. Further investigation of CdSe/ZnS QD with an amphiphilic/micelle coating has also found QDs to be present with skin capillaries in mice ${ }^{73}$. It was further reported by Larson et al. ${ }^{3}$, that the coating on QDs is pertinent to the mechanism of uptake, as well as the location of these NPs in cells. Clift et al. ${ }^{66}$ studied the uptake patterns and mechanisms of three different surface coated QDs. It was found that both the negatively and positively charged QDs entered J774.A1 macrophage cells via similar active pathways, resulting in their location within endosomes and eventually lysosomes. Additionally investigation of the uptake mechanisms of different surface coated/charged QDs has shown positively charged CdSe core/ZnS shell QDs to be present within the EL-4 cells outside of a membrane bound vesicle, however, $\mathrm{COOH}$ QDs were demonstrated to be present within endocytic vesicles ${ }^{59}$. It was also highlighted that the amine coated QDs were more susceptible to a reduction in stability over time, in comparison to the $\mathrm{COOH}$ QDs, which were subsequently observed to maintain their structure, possibly due to their location within endosomes ${ }^{59}$. It was concluded from these findings, that the presence of the positively charged QDs outside of a membrane-bound structure could be associated with the increased cytotoxicity observed with these QDs over the 12 hour period, also suggesting that a reduction in stability could be relative to QD toxicity. The findings of Hoshino et al. ${ }^{60}$ are in contrast to that of Clift et al.50, where it was shown that both negative and positively charged QDs $(\mathrm{COOH}$ and $\mathrm{NH}_{2}$ (PEG)) respectively) showed a decrease in their fluorescent intensity at $\mathrm{pH} 4.0$, indicative of a loss in their stability in this acidic environment (similar to that of an endosome/lysosome).

The suggestion that a reduction in QD stability is associated with their toxicity however, is in contrast to previous research by Hoshino et al.60 who showed uncoated CdSe/ZnS QDs, to be present within T-lymphoma tumour cells outside of membrane-bound vesicles, although maintained their stability within these tumour cells for up to two weeks, causing limited cytotoxicity. Furthermore, the suggestion that QDs reduce in stability is in contrast to $\mathrm{Xu}$ et al. ${ }^{74}$ who indicated that Gelatin and sodium dioctyl sulfosuccinate (AOT) coated CdSe QDs maintain their stability due to an enhancement of their band-edge luminescence over time in a series of environments, including light illumination.

The findings of these studies suggest that QD surface coating does have a significant effect on their uptake and cellular location and distribution. Problems are still paramount however, similar to those regarding gaining an understanding of the potentially adverse effects of QDs. Again, the use of completely different surface modified QDs is the main reason, but also the use of different cell types. Whilst this is also true for the biochemical effects, in regards to how QDs interact with cells, if the cells are immune cells (e.g. macrophages, or other cells expressing phagocytic properties) or not will have a profound impact upon how the QDs are observed to interact with these cells. It is clear therefore, that prior to the use of QDs in a clinical setting increased emphasis must be given towards understanding only those surface coatings, functionalisations and sizes (Fig. 2) that are intended for use in nanomedicine ${ }^{75}$. Despite this, initial investigation and use of QDs is already ongoing. 


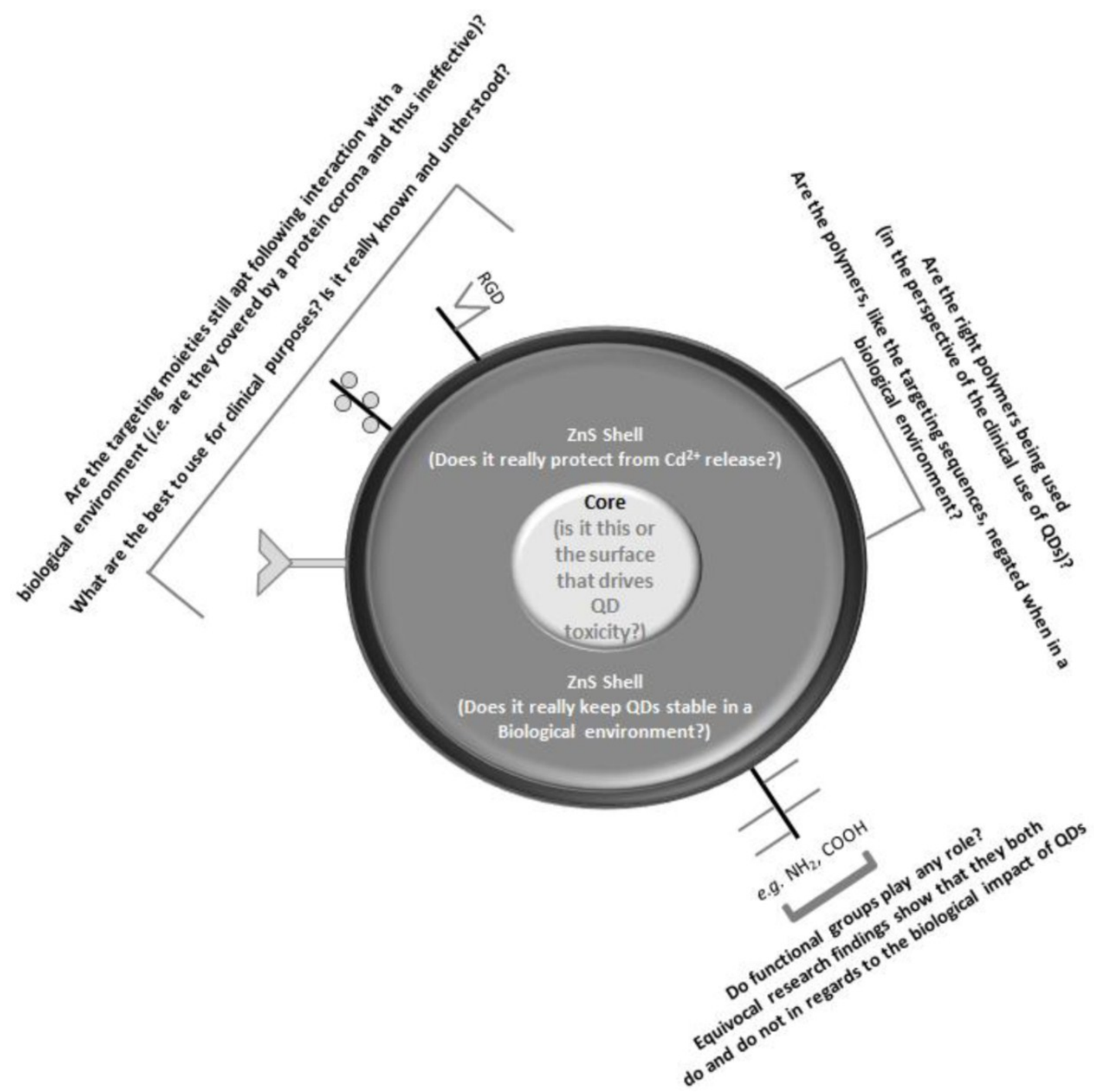

Figure 2: A perspective of how the different surface and physical characteristics are perceived and questioned in regards to the current research knowledge and understanding. A further discussion of how these aspects relate to the use of quantum dots in nanomedicine is also given.

\section{QDs as a Clinical Tool}

Although much debate surrounds the materials used to synthesize QDs, it is in fact the characteristics of these NPs that intrigue clinicians for their use within a medical setting. Due to their size, heightened applicability for surface medication and novel light emitting properties of QDs, which include a narrow emission spectra and increased photostability, these NPs have been increasingly used as advantageous alternative laboratory based bioimaging tools compared to organic fluorophores both in vitro and in vi$v 0^{76-84}$. It is these light emitting characteristics, specifically the ability of these NPs to emit light within the infrared spectrum; a wavelength of light capable to penetrate tissues and allow the visualisation of structures in vivo, such as tumours; that has enabled
QDs to be proposed as beneficial, non-invasive diagnostic imaging and therapeutic tools for the identification and treatment of disease in the human body $2,26,79,85$.

The advantages of using QDs as diagnostic tools were previously highlighted in a study by Gao et al. ${ }^{86}$, which examined the potential for cancer targeting using multifunctional NP probes based on QDs in vivo. It was reported that following both subcutaneous and systemic injection, the multifunctional QD probes efficiently accumulated within prostate tumours in vivo through the enhanced permeability and retention of the tumour sites, as well as the antibody binding of these NPs to the cancer-specific cell surface biomarkers. These findings support the conclusions of Wu et al. ${ }^{87}$ and Kim et al. ${ }^{88}$ that also stated conjugated 
and multifunctional QDs to be effective probes of cancer cells, and further advantageous diagnostic tools in vivo. Investigation using non-conjugated QDs has also found these semi-conductor NPs to be highly efficient in the tracing of cancer cells in vivo ${ }^{60}$. It was reported by Hoshino et al. ${ }^{60}$ that QDs were highly efficient in the targeting and fluorescence of T-lymphoma cells, further supporting the notion that QDs are beneficial diagnostic tools in vivo. The ability for QDs to act as novel fluorescent probes has most recently been shown by Gao and colleagues ${ }^{89}$. In this study, the authors synthesised QDs with dendrimers to form a novel 'drug delivery' system to identify and target acute tumours in vivo. It was observed that these QD-dendrimer formulations actively gained access to the tumour cells, whilst eliciting limited toxicity at the concentration tested (1nM) and maintaining their stability within a biological environment over a period of 10 weeks. This study further shows the clear benefits of QDs for diagnostic purposes and that with the correct surface modifications can elicit a limited level of adverse effects.

There are many studies within the literature that show QDs to be advantageous diagnostic tools in vitro and in vivo; thus ideal for use in a clinical setting. However, the predominant state of the literature shows that QDs, especially those with a Cd-based core, can elicit a heightened 'toxicity' that creates a sense that, in fact, QDs may not be an advantageous tool for nanomedicine. This however, is a common misinterpretation. As shown in the study by Gao et al. ${ }^{89}$, the particle concentration administered in vivo was $1 \mathrm{nM}$, whilst the concentration used within other in vivo and also in vitro studies are at least ten times higher ${ }^{51,64}$. Such a difference within the administered dose, independent of the biological system being used (although this most certainly plays a key role in the assessment of NP-biological interactions ${ }^{68}$ ), will more often than not elicit a (significantly) altered response. Therefore, as the debate continues within the field regarding 'realistic' concentrations (or more aptly 'doses') of NPs exposed to humans ${ }^{90}$, it is imperative that, concomitantly an understanding of how QDs interact with the entire human body is determined; in order to realise their potential clinical applicability. Recently, this has been taken a step further to reality by Ye and colleagues ${ }^{91}$. In a novel study, in which non-human primates (rhesus macques) were exposed via intravenous vein injection to chloroform dispersions of phospholipid-encapsulated micelle QDs at $25 \mathrm{mg} / \mathrm{kg}$ for up to three months (90 days). It was reported that after this chronic exposure period, no acute toxicity was associated with the QD exposure in any of the primates. It was stated however, that fol- lowing chemical analysis (inductively coupled mass spectroscopy) high levels of $\mathrm{Cd}$ were observed in the liver, spleen and kidneys after 90 days. It was suggested by the authors ${ }^{91}$ that the breakdown of the QDs was slow, and that the accumulation of such heavy metals in the major organs of the primates could impact upon their health and contribute to a deterioration of their life-span.

Although, as focussed upon within the present review, QDs are mainly identified as advantageous alternative diagnostic and therapeutic devices, as with many different nano-objects, QDs have also been suggested as potential drug delivery systems. Such a concept is thoroughly discussed and reviewed in the recent article by Ghaderi et al.92. However, in perspective, the use of QDs as a drug delivery tool within nanomedicine, whilst of course possible, is perhaps beyond the original perception of what QDs could offer to a clinical setting. Furthermore, with the many other potential drug delivery tools proposed for nanomedicine (of which the dendrimer is the prime example ${ }^{39}$ ) perhaps focussed concentration should be given to realising their diagnostic and therapeutic possibilities. Such possibilities will only be realised however, if clinical phase trials are conducted in an organised and collective manner. This will take time however, since the current literature and knowledge base regarding QD biological interactions is not sufficient. Although there is a plethora of research reports available, the inconsistencies are vast and have no collective, or correlation. It is therefore, important that clear, reproducible and representative biological systems are used ${ }^{93}$, as well as realistic QDs studied (i.e. those that are intended for use in nanomedicine and intentional human exposure). Furthermore, data regarding the actual use of QDs within a clinical setting is extremely limited due to (i) the unknown entity that QDs pose and (ii) the issue of ethical approval for their use within such a setting. It is essential therefore, that information to the first problem is gained with haste. As this will then provide clear evidence to overcome ethical issues and subsequently gain essential in vivo data concerning the applicability of QDs for use as a diagnostic and therapeutic tool within humans.

\section{Conclusion and outlook}

QDs elicit a number of novel physico-chemical characteristics that have allowed them to be considered as possible advantageous tools within nanomedicine. Despite the clear benefits proposed by QDs, heightened concerns have been raised as to their exposure to humans and what impact they might have upon human health. Increased research therefore has 
been performed in order to try and understand how QDs may interact with a variety of different in vivo and in vitro systems, and an increased knowledge base has been gained regarding this interaction and its subsequent biological effects. Despite this, limited information has been obtained that is useful towards understanding if QDs are applicable to a clinical setting in order to be used as either therapeutic or diagnostic tools. Predominantly, many of the studies have aimed to answer one specific scientific question (whether pertinent to human health, risk assessment or a comparison between QDs and another NP type). Although such information is essential to gain a clear understanding of how QDs interact with different biological systems, as clear overview, or outlook towards gaining an insight into how applicable QDs are for clinical use. It is essential therefore, for further research to be performed using a specific array of QDs with the same size, surface modifications and materials with a specific set of in vitro and in vivo systems that perfectly mimic the environment as found in the human body. Furthermore, the consequences of a prolonged QD exposure upon human health are not clear, and not well studied. Thus, consideration as to the chronic effects of QDs, as well as the impact of multiple exposures over time of QDs upon human health is paramount towards using these NPs within medical applications. Therefore, much research is still necessary in order to realise the potential of QDs as a tool within nanomedicine, but with the knowledge base already gained over the past two decades, both upon QD-biological system interactions and upon the $\mathrm{NP}$-cell interaction in general, it is possible to envisage the advantageous properties of these materials for the benefit of human health.

\section{Competing Interests}

The authors have declared that no competing interest exists.

\section{References}

1. ISO. International Organization for Standardization (ISO) Technical Specification (ISO/TS) 27687:2008; Nanotechnologies - Terminology and definitions for nano-objects - Nanoparticle, nanofibre and nanoplate; First published 2008-08-15. ISO. 2008.

2. Pison U, Welte T, Giersig M, Groneberg D.A. Nanomedicine for respiratory diseases. Eur J Pharm. 2006; 533: 341-350.

3. Mahmoudi M, Hofmann H, Rothen-Rutishauser B, Petri-Fink A. Assessing the in vitro and in vivo toxicity of superparamagnetic iron oxide nanoparticles. Chem Rev. 2012; 112: 2323-2338.

4. Hardman R. A toxicologic review of quantum dots: Toxicity depends on physiochemical and environmental factors. Environ Health Perspec. 2006; 114: 165-172.

5. Azzazy H.M.E, Mansour M.M.H, Kazmierczak S.C. From diagnostics to therapy: Prospects of quantum dots. Clin Biochem. 2007; 40: 917-927.

6. Pelly JL, Daar AS, Saner MA. State of academic knowledge on toxicity and biological fate of quantum dots. Toxicol Sci. 2009; 112: 276-296.

7. Maynard A.D. Nanotechnology: The next big thing, or much ado about nothing? Ann Occu Hyg.2007; 51: 1-12.
8. Müller L, Gasser M, Raemy D.O, Herzog F, Brandenberger C, Schmid O, Gehr P, Rothen-Rutishauser B, Clift M.J.D. Realistic exposure methods for investigating the interaction of nanoparticles with the lung at the air-liquid interface in vitro. InSci J (Nanotech). 2011; 1: 30-64.

9. Kreyling W.G, Hirn S, Schleh C. Nanoparticles in the lung. Nat Biotech. 2010; 28: 1275-1276

10. Johnston H.J, Semmler-Behnke M, Brown D.M, Kreyling W, Tran L, Stone V. Evaluating the uptake and intracellular fate of polystyrene nanoparticles by primary and hepatocyte cell lines in vitro. Toxicol Appl Pharm. 2010; 242: 66-78.

11. Geys J, De Vos R, Nemery B, Hoet P.H.M. In vitro translocation of quantum dots and influence of oxidative stress. Am J Physiol Lung Cell Mol Physiol. 2009; 297: L903-L911.

12. Oberdorster G, Stone V, Donaldson K. Toxicology of nanoparticles: A historical perspective. Nanotox. 2007; 1: 2-25

13. Ryman-Rasmussen J.P, Riviere J.E, Monteiro-Riviere N.A. Penetration of intact skin by quantum dots with diverse physicochemical properties. Toxicol Sci. 2006; 91: 159-165.

14. Ryman-Rasmussen J.P, Riviere J.E, Monteiro-Riviere N.A. Surface coatings determine cytotoxicity and irritation potential of quantum dot nanoparticles in epidermal kerationcytes. J Inves Derm. 2006; 127: 143-153.

15. Ryman-Rasmussen J.P, Riviere J.E, Monteiro-Riviere N.A. Variables influence interactions of untargeted quantum dot nanoparticles with skin cells an indentification of biochemical modulators. Nano Letts. 2007; 7: 1344-1348.

16. Wiesner M.R, Lowry G.V, Alvarez P, Dionysiou D, Biswas P. Assessing the risks of manufactured nanomaterials. Environ Sci Technol. 2006; 15: 4337-4345.

17. Summers H.D, Holton M.D, Rees P, Williams P.M, Thornton C.A. Analysis of quantum dot fluorescence stability in primary blood mononuclear cells. Cytom A. 2010; 77A: 933-939.

18. Rothen-Rutishauser B.M, Schuerch S, Haenni B, Kapp N, Gehr P. Interaction of fine particles and nanoparticles with red blood cells visualized with advanced microscopic techniques. Environ Sci Technol. 2006; 40: 4353-4359.

19. Alves E.N, PresgraveRde F, Presgrave O.A, Sabagh FP, de Freitas JC, Corrado A.P. A reassessment of the in vitro RBC haemolysis assay with defibrinated sheep blood for the determination of the ocular irritation potential of cosmetic products: comparison with the in vivo Draize rabbit test. Altern Lab Anim.2008; 36: 275-284.

20. Li X, Wang L, Fan Y, Feng Q, Cui F-Z. Biocompatibility and toxicity of nanoparticles and nanotubes. J Nanomat. 2012; doi:10.1155/2012/548389.

21. Lu S, Duffin R, Poland C, Daly P, Murphy F, Drost E, MacNee W, Stone V, Donaldson K. Efficacy of simple short-term in vitro assays for predicting the potential of metal oxide nanoparticles to cause pulmonary inflammation. Environ Health Perspec. 2009; 117: 241-247.

22. Zhu O, Wang Y, Zhou M, Mao C, Huang X, Bao J, Shen J. Preparation of anionic polyurethane nanoparticles and blood compatible behaviors. J Nanosci Nanotech. 2012; 12: 4051-4056.

23. Huang Z.W, Ding TT, Sun J. Study of Effect on Cell proliferation and Hemolysis of HAP and TCP Nanometer Particles. Adv Mater Res. 2011; 711: 378-379.

24. Shen M, Cai H, Wang X, Cao X, Li K, Wang SH, Guo R, Zheng L, Zhang $\mathrm{G}$, Shi X. Facile one-pot preparation, surface functionalization, and toxicity assay of APTS-coated iron oxide nanoparticles. Nanotech. 2012; 23: 105601.

25. Su, Y, Peng F, Jiang Z, Zhong Y, Lu Y, Jiang X, Huang Q, Fan C, Lee, S-T, He $Y$. In vivo distribution, pharmacokinetics, and toxicity of aqueous synthesized cadmium-containing quantum dots. Biomaterials. 2011; 32: 5855-5862.

26. Bailey R.E, Smith A.M, Nie S. Quantum dots in biology and medicine. Physica E. 2004; 25: 1-12.

27. Derfus A.M, Chan W.C.W, Bhatia S.N. Probing the cytotoxicity of semiconductor quantum dots. Nano Letts. 2004; 4: 11-18.

28. Walling MA, Novak JA, Shepard JRE. Quantum dots for live cell and in vivo imaging. Int J Mol Sci. 2009; 10: 441-491.

29. Tilley RD. Synthesis and applications of nanoparticles and quantum dots. Chem NZ. 2008;:146-150.

30. O'Farrell N, Houlton A, Horrocks BR. Silicon nanoparticles: applications in cell biology and medicine. Int J Nanomed. 2006; 1: 451-472.

31. Ferin J, Oberdorster G, Penney D.P. Pulmonary retention of ultrafine and fine particles in rats. Am J Respir Cell Mol Biol. 1992; 6: 535-542.

32. Oberdorster G, Oberdorster E, Oberdorster J. Nanotoxicology: An emerging discipline evolving from studies of ultrafine particles. Environ Health Perspec. 2005; 113: 823-839. 
33. Duffin R, Tran L, Brown D, Stone V, Donaldson K. Proinflammogenic effects of low-toxicity and metal nanoparticles in vivo and in vitro: Highlighting the role of particle surface area and surface reactivity. Inhal Toxicol. 2007; 19: 849-856.

34. Bouwmeester H, Lynch I, Marvin H.J.P, Dawson K.A, Berges M, Braguer D, Byrne H.J, Casey A, Chambers G, Clift M.J.D, Elia G, Fernandes T.F, Fjellsbø L.B, Hatto P, Juillerat L, Klein C, Kreyling W.G, Nickel C, Riediker M, Stone V. Minimal analytical characterisation of engineered nanomaterials needed for hazard assessment in biological matrices. Nanotox. 2011; 5: 1-11.

35. Nel A.E, Maedler L, Velegol D, Xia T, Hoek E.M.V, Somasundaran P, Klaessig F, Castranova V, Thompson M. Understanding biophysicochemical interactions at the nano-bio interaface. Nat Mater. 2009; 8: 543-557.

36. Lynch I, Dawson K.A. Protein-nanoparticle interactions. Nanotoday. 2008; 3: 40-47.

37. Monopoli M.P, Walczyk D, Campbell A, Elia G, Lynch I, Bombelli F.B, Dawson K.A. Physical-Chemical aspects of protein corona: Relevance to in vitro and in vivo biological impacts of nanoparticles. JACS. 2011; 133: 2525-2534.

38. Wick P, Manser P, Limbach L.K, Dettlaff-Weglikowska U, Krumeich F, Roth S, Stark W.J, Bruinink A. The degree and kind of agglomeration affect carbon nanotube cytotoxicity. Tox Letts. 2007; 168: 121-131.

39. Duncan R. The dawning era of polymer therapeutics. Nat Drug Discov. 2003; 2: 347-360.

40. Moghimi S.M, Szebeni J. Stealth liposomes and long circulating nanoparticles: critical issues in pharmacokinetics, opsonization and protein-binding properties. Pro Lipid Res. 2003; 42: 463-478.

41. Moghimi SM. The effect of methoxy-PEG chain length and molecular architecture on lymph node targeting of immuno-PEG liposomes. Biomaterials. 2006; 27: 136 - 144.

42. Porter C.J.H, Moghimi S.M, Illum L, Davis S.S. The polyoxyethylene/polyoxyproplylene block co-polymer Poloxamer-407 selectiely redirects intravenously injected microspheres to sinusoidal dothelial cells of rabbit bone marrow. FEBS Letts. 1992; 305: 62-66.

43. Gref R, Minamitake Y, Peracchia M.T, Trubetskoy V, Torchilin V, Langer R. Biodegradable long-circulating polymeric nanospheres. Science. 1994; 263: 1600-1603.

44. Storm G, Belliot S.O, Daemen T, Lasic D.D. Surface modification of nanoparticles to oppose uptake by the mononuclear phagocyte system. Adv Drug Del Rev. 1995; 17: 31-48.

45. Peracchia M.T, Fattal E, Desmaele D, Besnard M, Noel J.P, Gomis J.M. Stealth PEGylated polycyanoacrylate nanoparticles. Eur J Pharm Biopharm. 1999; 58: 327-341.

46. Hayes M.E, Drummond D.C, Hong K, Zheng W.W, Khorosheva V.A, Cohen J.A, Noble IV C.O, Park J.W, Marks J.D, Benz C.C, Kirpotin D.B. Increased target specificity of Anti-HER2 genospheres by modification of surface charge and degree of PEGlyation. Mol Pharm. 2006; 3: 726-736.

47. Veronese F.M, Pasut G. PEGylation, successful approach to drug delivery. Drug Discovery Today. 2005; 10: 1451-1458.

48. Zhang T, Stilwell J.L, Gerion D, Ding L, Elboudwarej O, Cooke P.A, Gray J.W, Alivisatos A.P, Chen F.F. Cellular effect of high doses of silica-coated quantum dot profiled with high throughput gene expression analysis and high content cellomics measurements. Nano Letts. 2006; 6: 800-808.

49. Duan H, Nie S. Cell-penetrating quantum dots based on multivalent and endosome-disrupting surface coatings. JACS. 2007; 129: 3333-3338.

50. Clift M.J.D, Rothen-Rutishauser B, Brown D.M, Duffin R, Donaldson K, Proudfoot L, Guy K, Stone V. The Impact of Different Nanoparticle Surface Chemistry and Size on Uptake and Toxicity in a Murine Macrophage Cell Line. Toxicol Appl Pharm. 2008; 232: 418-427.

51. Clift M.J.D, Varet J, Hankin S.M, Brownlee B, Davidson A, Brandenberger C, Rothen-Rutishauser B, Brown D.M, Stone V. Quantum dot cytotoxicity in vitro: An investigation into the cytotoxic effects of a series of different surface chemistries and their core/shell materials. Nanotox. 2011; 5: 664-674.

52. Clift M.J.D, Boyles M.S.P, Brown D.M, Stone V. An investigation into the potential for different surface coated quantum dots to cause oxidative stress and affect macrophage cell signalling in vitro. Nanotox. 2010; 4: 139-149.

53. World Health Organisation. International Programme on Chemical Safety; Environmental Health Criteria (EHC) 134; Cadmium. WHO. 1992

54. Stohs S.J, Bagchi D. Oxidative mechanisms in the toxicity of Metal Ions. Free Rad Biol Med. 1995; 18: 321-336.

55. Belyaeva E.A, Korotkov S.M. Mechanism of primary Cd2+-induced rat liver mitochondria dysfunction: discrete modes of $\mathrm{Cd} 2+$ action on cal- cium and thiol-dependent domains. Toxicol Appl Pharm. 2003; 192: 56-68.

56. Stohs S.J, Bagchi D, Hassoun E, Bagchi M. Oxidative mechanisms in the toxicity of chromium and cadmium ions. J Environ Path Toxicol Oncol. 2001; 20: 77-88.

57. Sadler P.J, Viles J.H. $1 \mathrm{H}$ and $113 \mathrm{C}$ NMR Investigations of $\mathrm{Cd} 2+$ and $\mathrm{Zn} 2+$ Binding Sites on Serum Albumin: Competition with $\mathrm{Ca} 2+, \mathrm{Ni} 2+, \mathrm{Cu} 2+$, and Zn2+. Inorg Chem. 1996; 35: 4490-4496.

58. Shiohara A, Hoshino A, Hanaki K, Suzuki K, Yamamoto K. On the cyto-toxicity caused by quantum dots. Microbiol Immunol. 2004; 48: 669-675.

59. Hoshino A, Fujioka K, Oku T, Masakazu S, Sasaki Y.F, Ohta T, Yasuhara M, Suzuki K, Yamamoto K. Physicochemical properties and cellular toxicity of nanocrystal quantum dots depend on their surface modification. Nano Letts. 2004; 4: 2163-2169.

60. Hoshino A, Hanaki K, Suzuki K, Yamamoto K. Applications of T-lymphoma labelled with fluorescent quantum dots to cell tracing markers in mouse body. Biochem Biophys Res Comm. 2004; 314: 46-53.

61. Chan W, Shiao N, Lu P. CdSe quantum dots induce apoptosis in human neuroblastoma cells via mitochondrial-dependent pathways and inhibition of survival signals. Tox Letts. 2006; 167: 191-200.

62. Guo G, Liu W, Liang J, He Z, Xu H, Yang X. Probing the cytotoxicity of CdSe quantum dots with surface modification. Mater Lett. 2007; 61:1641-1644.

63. Lovric J, Bazzi H.S, Cuie Y, Fortin G.R.A, Winnik F.M, Maysinger D. Differences in subcellular distribution and toxicity of green and red emitting CdTe quantum dots. J Mol Med. 2005; 83: 377-385.

64. Geys J, Nemmar A, Verbeken E, Smolders E, Ratoi M, Hoylaerts MF, Nemery B, Hoet PHM. Acute toxicity and prothrombotic effects of quantum dots: Impact of surface charge. Environ Health Perspec. 2008; 116: 1607-1613.

65. Hoshino A, Hanada S, Yamamoto K. Toxicity of nanocrystal quantum dots: the relevance of surface modifications. Arch Toxicol. 2011; 85: 707-720.

66. Clift M.J.D, Brandenberger C, Rothen-Rutishauser B, Brown D.M, Stone $\mathrm{V}$. The uptake and intracellular fate of a series of different surface coated quantum dots in vitro. Toxicol. 2011; 286: 58-68.

67. Lovric J, Cho S.J, Winnik F.M, Maysinger D. Unmodified cadmium telluride quantum dots induce reactive oxygen species formation leading to multiple organelle damage and cell death. Chem Biol. 2005; 12: 1-8.

68. Clift M.J.D, Gehr P. Rothen-Rutishauser B. In vitro Testing for Nanotoxicology: A valid alternative? Arch Toxicol. 2011; 85: 723-731.

69. Maysinger D, Lovric J, Eisenberg A, Savic R. Fate of micelles and quantum dots in cells. Eur J Pharm Biopharm.2007; 65:270-281.

70. Zhang L.W, Monteiro-Riviere N.A. Mechanisms of quantum dot nanoparticle cellular uptake. Toxicol Sci. 2009; 110: 138-155.

71. Jaiswal J.K, Mattoussi H, Mauro J.M, Simon S.M. Long-term multiple colour imaging of live cells using quantum dot bioconjugates. Nat Biotech. 2003; 21: 47-51.

72. Dubertret B, Skourides P, Norris D.J, Noireaux V, Brivanlou A.H, Libchaber A. In vivo imaging quantum dots encapsulated in phospholipids micelles. Sci. 2002; 289: 1759-1762.

73. Larson D.R, Zipfel W.R, Williams R.M, Clark S.W, Bruchez M.P, Wise F.W. Water-soluble quantum dots for multiphoton fluorescence imaging in vivo. Sci. 2003; 300: 1434-1436.

74. Xu L, Chen K, El-Khair H.M, Li M, Huang X. Enhancement of band-edge luminescence and photo-stability in colloidal CdSe quantum tods by various surface passivation technologies. Appl Surf Sci. 2001; 172: 84-88.

75. Mout R, Moyano DF, Rana S, Rotello VM. Suface functionalization of nanoparticles for nanomedicine. Chem Soc Rev. 2012; 41: 2539-2544.

76. Parak W.J, Boudreau R, LeGros M, Gerion D, Zanchet D, Micheel C.M. Cell motility and metastatic potential studies based on quantum dot imaging of phagokineitc tracks. Adv Mater. 2002; 14: 882-885.

77. Ness J.M, Akhtar R.S, Latham C.B, Roth K.A. Combined tyramide Signal amplification and quantum dots for sensitive and photostable immunofluorescence detection. J Histochem Cytochem. 2003; 51: 981-987.

78. Lidke D.S, Nagy P, Heintzmann R, Arndt-Jovin D.J, Post J.N, Grecco H.E, Jares-Erijman E.A, Jovin T.M. Quantum dot ligands provide new insights into erbB/HER receptor-mediated signal transduction. Nat Biotech. 2004; 22: 198-203.

79. Nisman R, Dellaire G, Ren Y, Li R, Bazett-Jones D.P. Application of quantum dots as probes of correlative fluorescence, conventional, and energy-filtered transmission electron microscopy. J Histochem Cytochem. 2004; 52: 13-18.

80. Gao X, Yang L, Petro J.A, Marshall F.F, Simons J.W, Nie S. In vivo molecular and cellular imaging with quantum dots. Cur Opin Biotech. 2005; 16: 63-72. 
81. Giepmans B.N.G, Deerinck T.J, Smarr B.L, Jones Y.Z, Ellisman M.H. Correlated light and electron microscopic imaging of multiple endogenous proteins using quantum dots. Nat Meth. 2005; 2: 743-749.

82. Kloepfer J.A, Mielke R.E, Nadeau J.L. Uptake of CdSe and CdSe/ZnS quantum dots into bacteria via purine-dependent mechanisms. Appl Environ Microbiol. 2005; 71: 2548-2557.

83. Mason J.N, Farmer H, Tomlinson I.D, Schwartz J.W, Savchenko V, DeFelice L.J, Rosenthal S.J, Blakely R.D. Novel fluorescence based approaches for the study of biogenic amine transporter localization, activity and regulation. J Neurosci Meth. 2005; 143: 3-25.

84. Pinaud F, Michalet X, Bentolila L.A, Tsay J.M, Doose S, Li J.J, Iyer G, Weiss S. Advances in fluorescence imaging with quantum dot bio-probes. Biomaterials. 2006; 27: 1679-1687.

85. Michalet X, Pinaud F.F, Bentolila L.A, Tsay J.M, Doose S, Li J.J, Sundaresan G, Wu A.M, Gambhir S.S, Weiss S. Quantum dots for live cells, in vivo imaging, and diagnostics. Science. 2005; 307: 538-544.

86. Gao X, Cui Y, Levenson R.M, Chung L.W.K, Nie S. In vivo cancer targeting and imaging with semiconductor quantum dots. Nat Biotech. 2004; 22: 969-976.

87. Wu X, Liu H, Liu J, Haley K.N, Treadway J.A, Larson J.P, Ge N, Peale F, Bruchez M.P. Immunofluorescent labelling of cancer marker Her2 and other cellular targets with semiconductor quantum dots. Nat Biotech. 2003; 21: 41-46.

88. Kim S, Lim Y.T, Soltesz E.G, De Grand A.M, Lee J, Nakayama A, Parker J.A, Mihalejevic T, Laurence R.G, Dor D.M, Chon O.L.H, Bawedni M.G, Frangioni J.V. Near-infrared fluorescent type II quantum dots for sentinel lymph node mapping. Nat Biotech. 2004; 22: 93-97.

89. Gao J, Chen K, Luong R, et al. A novel clinically translatable flurescent nanoparticle for targeted molecular imaging of tumors in living subjects. Nano Letts. 2012; 12: 281-286.

90. Oberdorster G. Safety assessment for nanotechnology and nanomedicine: concepts of nanotoxicology. J Int Med. 2010; 267: 89-105.

91. Ye L, Yong K-T, Liu L, et al. A pilot study in non-human primates shows no adverse response to intravenous injection of quantum dots. Nat Nanotech. 2012; DOI: 10.1038/NNANO.2012.74

92. Ghaderi S, Ramesh B, Seifalian AM. Fluorescence nanoparticles "quantum dots" as drug delivery system and their toxicity: a review. J Drug Target. 2011; 19: 475-486.

93. Rothen-Rutishauser B, Blank F, Muehlfeld C, Gehr P. In vitro models of the human epithelial airway barrier to study the toxic potential of particulate matter. Exp Opin Drug Metab Toxicol. 2008; 4: 1075-1089. 